\title{
Size, geometry and nonuniformity effects of surface-nanocrystalline aluminum in nanoindentation test
}

\author{
Yueguang Wei *, Siqi Shu, Ying Du, Chen Zhu \\ LNM, Institute of Mechanics, Chinese Academy of Sciences, Beijing 100080, PR China \\ Received 8 March 2004 \\ Available online 27 June 2005
}

\begin{abstract}
In the present research, the mechanical behavior of the surface-nanocrystalline aluminum (SNCA) is investigated through nanoindentation experiment and theoretical modeling. Firstly, through microscopical observation and measurement for the SNCA material, a microstructure cell model is developed. Secondly, based on the microstructure cell model and the strain gradient plasticity theory, and based on introducing a parameter accounting for the grain size nonuniformity effect, the discrete features of the hardness-depth relations of the SNCA material are described. The "U-type" feature of the hardness-depth experimental curves is modeled and simulated. Thirdly, in the SNCA material the mechanical property of the grain boundary, i.e., the strength of plastic zone penetrating the grain boundary is characterized by introducing a criterion parameter, the critical effective plastic strain. The "waterfall-type" feature of the hardnessdepth curves is modeled and simulated. It is worth pointing out that, in the present study, the length scale parameter in the strain gradient plasticity theory is taken as a universal material parameter instead of a simulation parameter, and it is determined through applying the strain gradient plasticity theory to the modeling of the corresponding single crystal aluminum.
\end{abstract}

(C) 2005 Elsevier Ltd. All rights reserved.

Keywords: Surface-nanocrystalline material; Microstructure cell model; Size effect; Geometrical effect; Strain gradient plasticity; Nanoindentation test

\footnotetext{
${ }^{*}$ Corresponding author. Fax: +86106256 1284.

E-mail address: Ywei@LNM.imech.ac.cn (Y. Wei).
}

0749-6419/\$ - see front matter @ 2005 Elsevier Ltd. All rights reserved. 


\section{Introduction}

Resent studies have shown that the high strength nano-structured materials can be manufactured by using some advanced techniques, and on the other hand the mechanical behavior of the conventional materials can be improved by using some surfacenanocrystalline techniques. For example, severe plastic deformation (SPD) method is used to manufacture the nanocrystalline (NC) metals (see Valiev et al., 1993, 1996, 1999, 2000; Lu and Lu, 1999). The SPD methods mainly include: the large torsion method (Valiev et al., 1993), the large pressing method (Valiev et al., 2000) and the ultrasonic shot peening (USP) method ( $\mathrm{Lu}$ and Lu, 1999), etc. Through microscopical observation using the TEM technique, Wu et al. (2002) and Zhu (2002) have displayed the microstructure features of both the nano-structured bulk materials and the surface-nanocrystalline materials, which are of the regular microstructures overall or locally. The microstructure cell size usually is from tens to hundreds of nanometers, even to microns. Within this length scale, solid often behaves a strong size effect (Fleck et al., 1994; Ma and Clarke, 1995; Stolken and Evans, 1998; McElhaney et al., 1998; Xue et al., 2002; Huang et al., 2004). About the size effect study, in fact many investigations were focused on the nanoindentation tests for single crystal metals, and most results showed that as the indent depth decreases, the measured hardness curve displays an obviously increasing trend, i.e., indentation size effect. The size effects were often described by using the strain gradient theories (Aifantis, 1984; Fleck and Hutchinson, 1997; Wei and Hutchinson, 1997; Begley and Hutchinson, 1998; Gao et al., 1999; Acharya and Bassani, 2000; Chen and Wang, 2000, 2002; Wei, 2001; Wei et al., 2001; Wei and Hutchinson, 2003; Hwang et al., 2003; Huang et al., 2004; Abu Al-Rub and Voyiadjis, 2004; etc.), and to some extent most simulation results were consistent with the experimental data. Besides the strain gradient theory models, other models were also found in describing the size effect, such as, a model based on a surface work and plastic volume work concept (Horstemeyer et al., 2001; Gerberich et al., 2002), and a homogenization model for the damage (Taylor et al., 2002; Saczuk et al., 2003; Bonfoh et al., 2004; Brunig and Ricci, 2005), etc. About using the strain gradient theory models to study the size effects, previous studies have shown that the microscale parameter in the strain gradient theory is of a micron order for single crystal metals (Wei et al., 2001, 2003; etc.). However, for the nanocrystalline material the concept of the size effect is very complicated, about which besides the size effect mentioned above, the influences of the crystal grain size, grain shape distributions, and the deviation of crystal grain sizes from local uniformity on the material behaviors should be considered. In the author's previous research on the nanocrystalline Al-alloy and the thin film/substrate system (Wei et al., 2003, 2004a), the effect from both the crystal grain size and the grain shape distribution was called the geometrical effect in order to differ from the size effect. In the present research, we simply call the effect due to deviation of crystal grain sizes from local uniformity as the grain size nonuniformity effect. This effect will be considered in our microstructure cell models.

Until recently, only few studies have been undertaken on the mechanical behavior of the surface-nanocrystalline materials. Wu et al. (2002) and Zhu (2002) carried out the TEM microscopy analysis for the microstructure features. The nanocrystal 
mechanisms were interpreted from dislocation density and in the submicron region motion. Based on the molecular dynamics simulation a functional relationship between the dislocation density and the nanocrystal grain size was obtained (Ma et al., 2003). Wei and others used the microstructure cell model and strain gradient theory to study the size and geometrical effects of a nanocrystalline Al-alloy under compression and nanoindentation (Wei et al., 2004a). In the present research, the mechanical behavior of a surface-nanocrystalline aluminum (SNCA) obtained by the USP method ( $\mathrm{Lu}$ and $\mathrm{Lu}, 1999$ ) will be studied using microscopy observation, experimental measurement and model analyses. In the experimental research, the specimens will be designed and prepared according to the observed microstructure features. Nanoindentation experiments will be carried out based on both the continuous stiffness method and the randomly selected loading point method. The corresponding microstructure cell models put forwarded by Wei et al. (2003, 2004a) will be developed to consider the grain size nonuniformity effect. Strain gradient plasticity theory will be used to simulate the nanoindentation experiments. The effects of the length parameter in the strain gradient theory (size effect), of the grain sizes and their shapes (geometrical effect), as well as of the grain size nonuniformity are simultaneously considered. The characteristics of the indenter or plastic zone penetrating the grain boundaries will be simulated. Through comparison of experimental data with theoretical predictions, both material parameters and the model parameters will be attained.

\section{Microscopy observation and measurement for the SNCA material}

First, through microscopy observation and analysis for the SNCA material, the microstructure features will be discussed. Second, the nanoindentation experiments are performed by using both the continuous stiffness method and the randomly selected load point method.

\subsection{Microscopy observation and analysis}

Fig. 1(a) shows a schematic illustration of the nanocrystallization SPD method ( $\mathrm{Lu}$ and $\mathrm{Lu}, 1999)$. When a material free surface is continuously subjected to the ultrasonic shot peening (USP), nanometer-sized crystals are generated within a thin layer near the NC surface. As shown in Fig. 1(b) and (c), the grain size gradually increases toward the interior of the material reaching the initial size at about $100 \mu \mathrm{m}$. Fig. 1(c) is a plot of the averaged grain size as a function of distance $(D)$ along a direction toward the interior of the material from the NC surface. Note that, the horizontal coordinate scale is of two order magnitude larger than that of the corresponding vertical coordinate scale. From Fig. 1(c), at a given distance $D$ the statistically averaged grain size is not uniform. Since the nanoindentation test result is very sensitive to local features of the specimen surface, it is very important and interesting to study the grain size nonuniformity influence on the nanoindentation test results. 
a

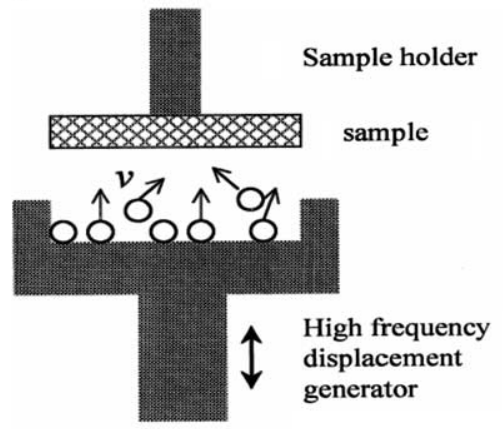

c
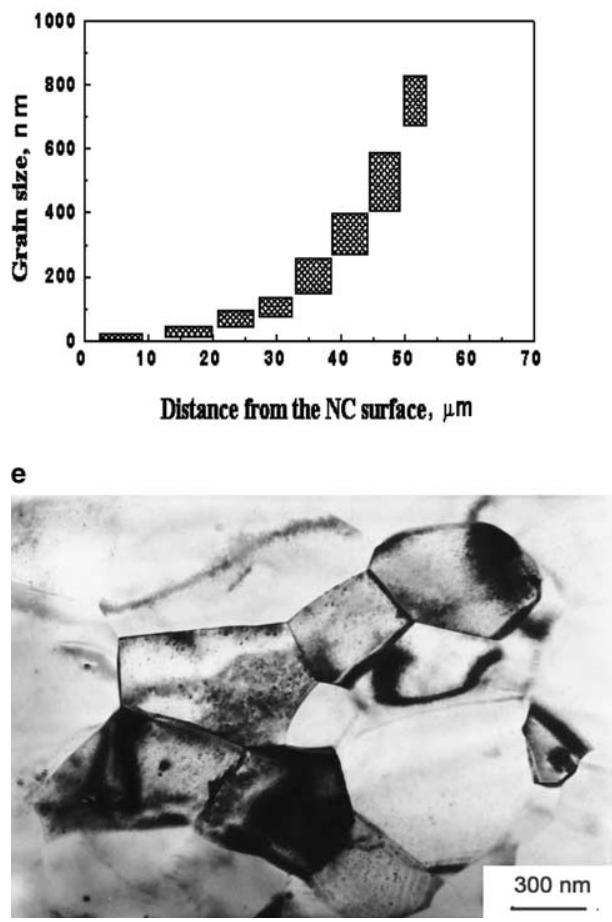

b

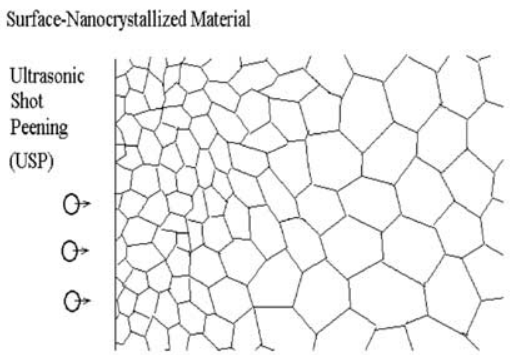

d

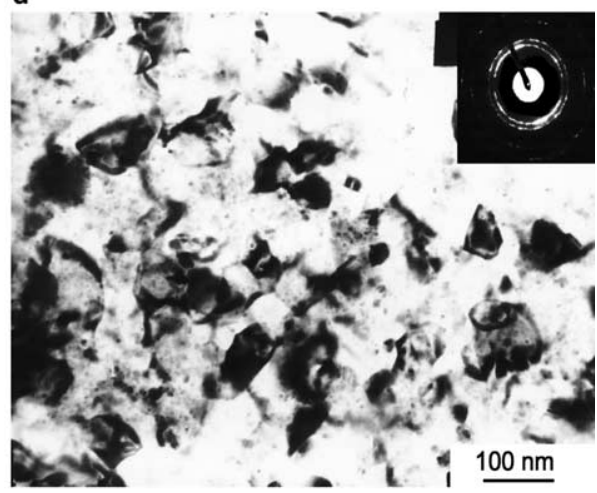

f

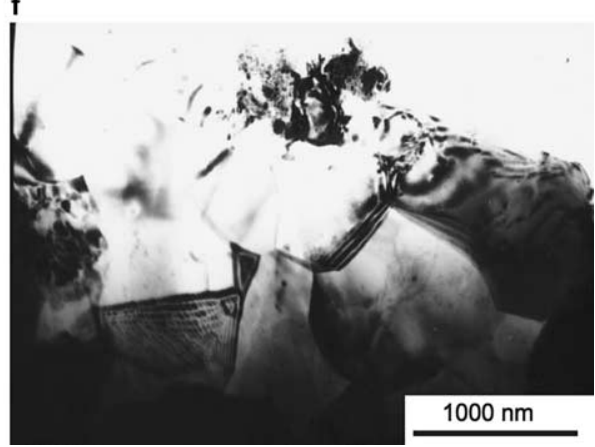

Fig. 1. Sketches of surface-nanocrystallization technique (a) and (b), grain size distribution along the normal direction of the NC surface ( $t-D$ relation) (c), and the TEM photos about the formed nanometersized grains (d)-(f), where (d) for $D=15 \mu \mathrm{m}$, (e) for $D=40 \mu \mathrm{m}$ and (f) for $D=55 \mu \mathrm{m}$.

TEM photos of the higher-resolution electron microscopy for the SNCA material produced by USP method are shown in Fig. 1(d)-(f). The fabrication process was performed at the Metal Institute, Chinese Academy of Sciences (Zhu, 2002). The material microstructures observed on the cast surfaces, parallel to the NC surface for $D=15,40$ and $55 \mu \mathrm{m}$, are shown in Fig. 1(d), (e) and (f), respectively. 
A regular nanocrystal structure within a layer near the NC surface is formed after nanocrystallization, and the mean grain size $(t)$ increases with $D$. The nanocrystal grain shape along the direction normal to the NC surface is somewhat flat from microscopic observation. At $D=15 \mu \mathrm{m}$ (Fig. 1(d)), nanometer-sized crystal grains are observed. From Figs. 1(e) and (f) the submicron grains and micron scale grains can be observed. From Fig. 1(d)-(f), for a series of distances away from the NC surface, clearly the grain sizes are not uniform. The uniformity deviation (grain size nonuniformity effect) may considerably influence the experimental measurement data in nanoindentation test. Fig. 1(d)-(f) show the formed grains at $D=15,40$ and $55 \mu \mathrm{m}$, respectively. In contrast to the conventional polycrystal material, nanocrystal grain boundary is with a given thickness and with a thin layer microstructure (Wei et al., 2004a).

\subsection{Nanoindentation tests}

Nanoindentation experiments are carried out at the State Key Laboratory of Friction and Lubrication, at Tsinghua University, and at the Metal Institute, Chinese Academy of Sciences, respectively. The instrument of the former is a CSM-Nanoindenter (made in Switz). The nanoindentation test method is the randomly selected load point method. The instrument of the latter is an MTS-Nanoindenter-XP. The nanoindentation test method is the continuous stiffness method, i.e., the hardnessdepth curve is continuously obtained during indentation at a fixed loading point. Indenter tip shapes for both instruments are identical, Berkovich tip with a $40 \mathrm{~nm}$ tip radius.

Nanoindentation experiment was carried out on specimen surface paralleling to NC surface approximately at $D=25 \mu \mathrm{m}$, that is, the grain size ranges from 50 to $150 \mathrm{~nm}$. In the present experiment, due to the grain size nonuniformity effect, initially, the indenter tip may be located at either large size grain region or the small size grain region. When horizontal size of contact zone is smaller than the horizontal size of the indented grain, or when the vertical size of the contact zone is smaller than the vertical size of the indented grain, the plastic zone will be constrained within the grain region when grain boundary constraint strength is high enough, otherwise, the plastic zone will be extended to the neighboring grain region.

\subsubsection{Nanoindentation with the continuous stiffness method}

Fig. 2(a)-(c) show the load-contact depth curves and the hardness-depth curves for 6 different indenting locations at a same distance from the NC surface, $D=25 \mu \mathrm{m}$. The load-depth curves seem to be insensitive to the loading positions, while hardness-depth curves are significantly dependent upon the loading points, especially at shallow indentation depths. This may be due to the grain size nonuniformity effect. From Fig. 2(b) or (c), given a distance $D$, the hardness values of the SNCA material are taken from a discrete strip. This dependence of hardness on grain size nonuniformity was treated from a viewpoint of surface roughness by Wei et al. (2004b). A specific hardness-depth experimental data (for a given loading point) is 

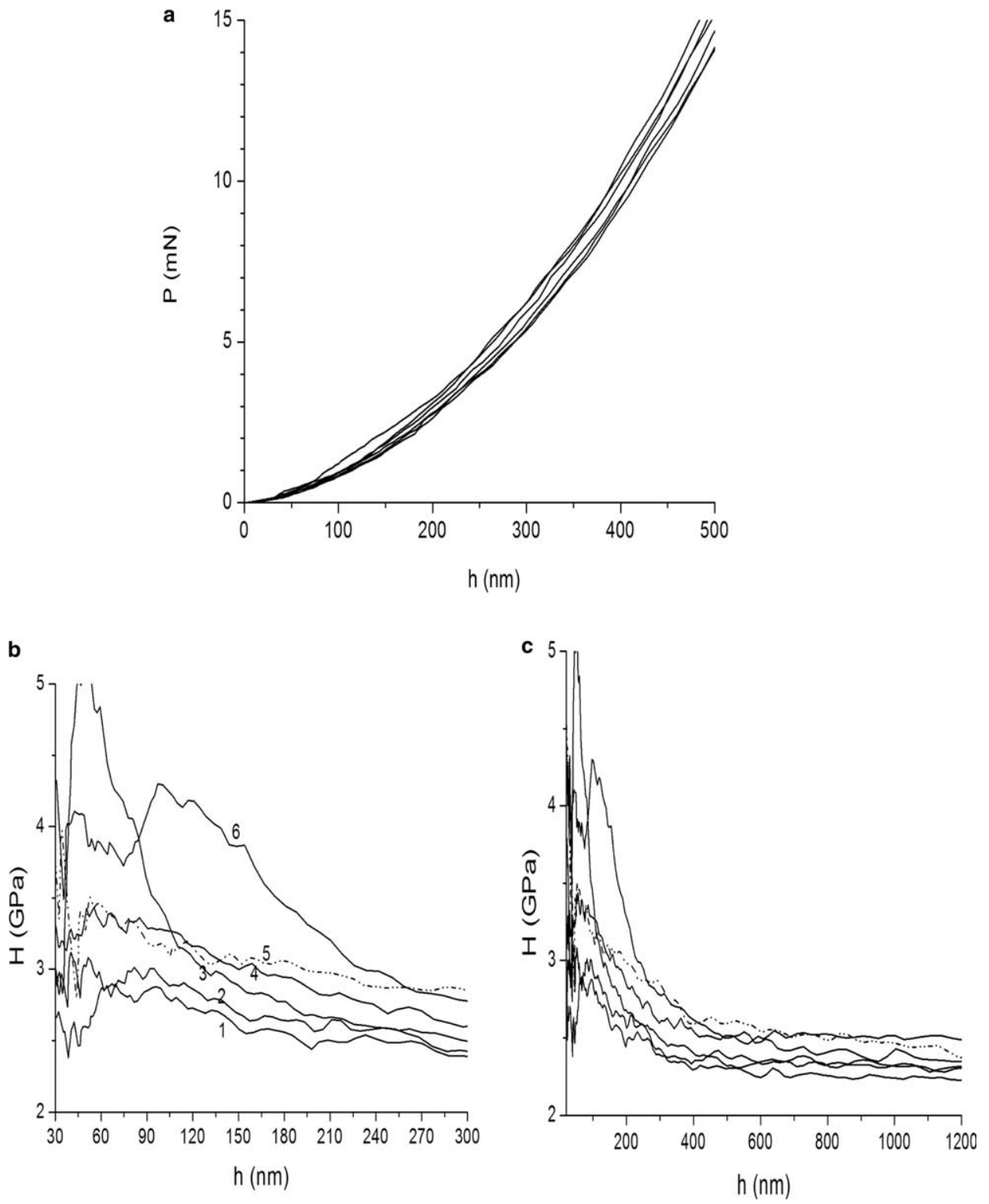

Fig. 2. Nanoindentation experimental results based on the continuous stiffness method at the distance $D=25 \mu \mathrm{m}$ (away from the NC surface). Load-indent depth curves (a), hardness-indent depth curves for small horizontal coordinate scale (b) and for large horizontal coordinate scale (c). Six load point results are given in the figure.

shown in Fig. 3(a) and (b). The experimental data curves are not smooth due to the grain boundary constraints on the plastic zone and due to the plastic zone penetrating the grain boundary. 

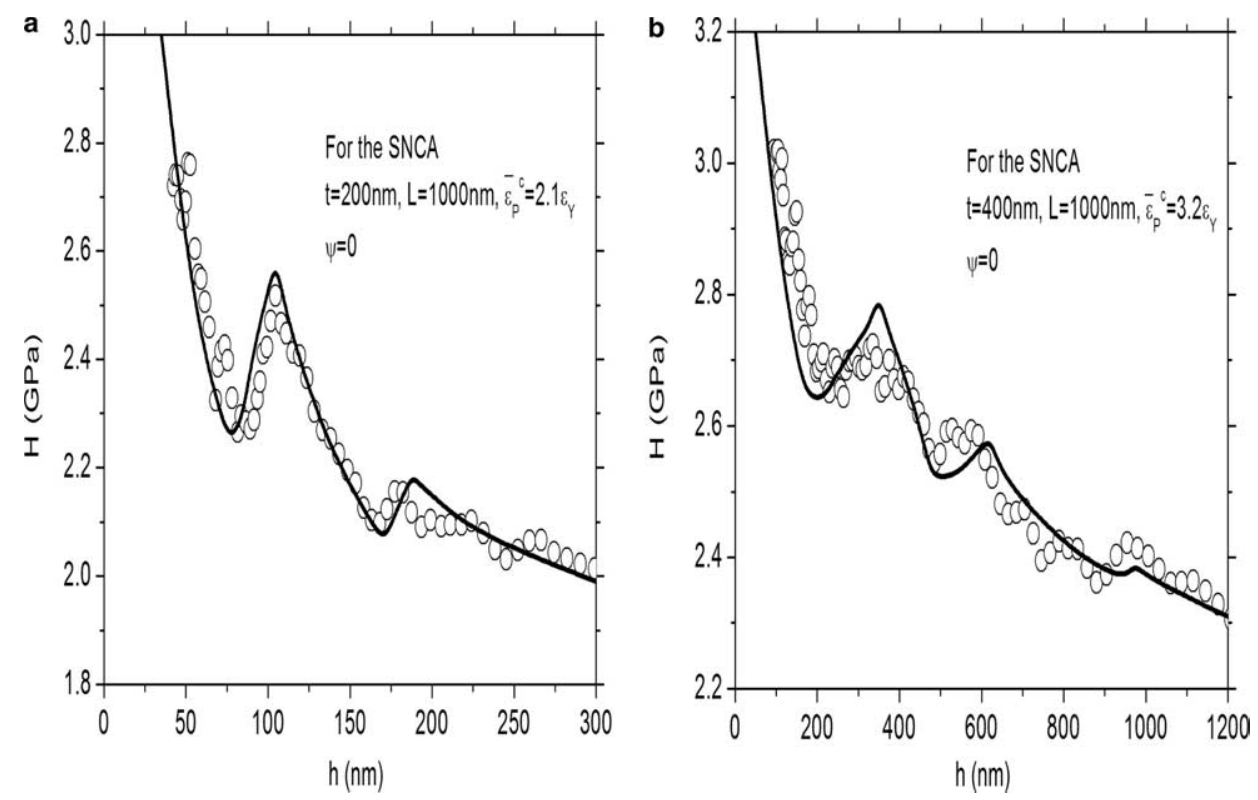

Fig. 3. Hardness-depth experimental data for the SNCA material based on the continuous stiffness method. (a) and (b) correspond to two load points at $D=25 \mu \mathrm{m}$. The theoretical modeling results (solid lines) are also shown in the figures, where the grain sizes are taken as $t=200 \mathrm{~nm}$ for case (a) and $t=400 \mathrm{~nm}$ for case (b), the other parameter values have shown in figures.

\subsubsection{Nanoindentation with the randomly selected loading point method}

By randomly selecting the loading points at $D=50,60$ and $70 \mu \mathrm{m}$ away from the NC surface, respectively (corresponding the average grain sizes are 600, 1000, $2000 \mathrm{~nm}$, respectively), and carrying out the nanoindentation experiments, the obtained hardness-depth data are shown in Fig. 4(a)-(c). Note that the maximum value of indent depth here for each case is controlled smaller than the grain sizes of corresponding indent point. A trend of the hardness-depth data, a "U-type" characteristic for each case is assumed. When indent depth is small, the hardness value decreases with increasing indent depth (i.e., size effect feature), then goes down through a minimum value. The hardness increases with further increasing the indent depth due to plastic zone constrained by grain boundaries (geometrical effect). The hardness-depth data are always a discrete strip due to the grain size nonuniformity effect. The corresponding depth locations of the minimum points of hardness data depend on the average grain sizes. Prior to the minimum point, the size effect dominates. After that the geometrical effect dominates. For comparison, Fig. 4(d) shows the hardness-depth experimental data for single crystal aluminum based on the randomly selecting load point method. From Fig. 4(d), a trend of the hardness-depth experimental data, an "L-type" feature can be observed, and the size effect dominates. This feature can be modeled based on the strain gradient plasticity theory, and by comparing the modeling result with experimental data curve the length scale parameter in the strain gradient theory can be determined for aluminum. Through 

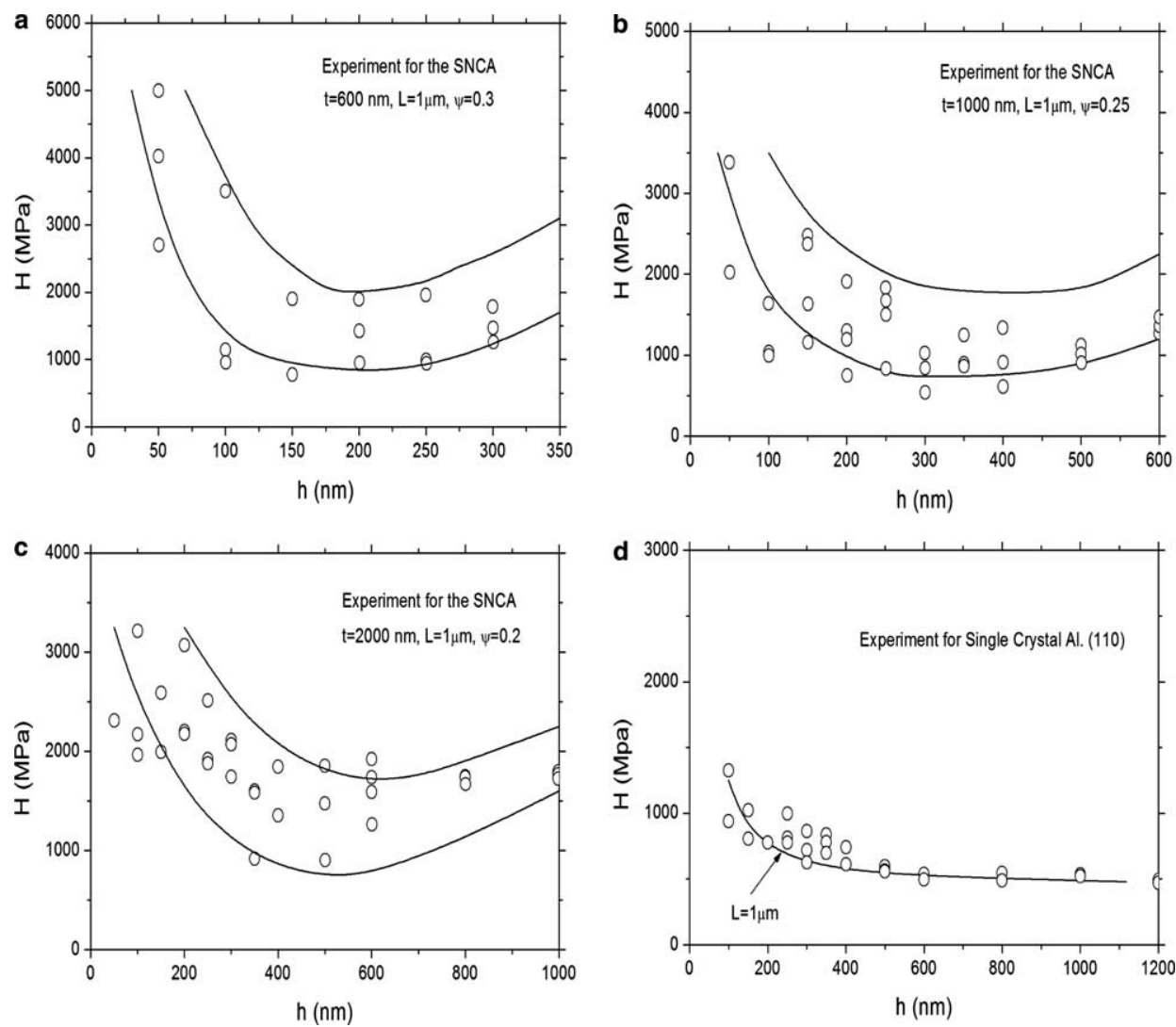

Fig. 4. Hardness-indent depth relation data for the SNCA material based on the randomly selected load point method. (a)-(c) correspond to different grain sizes $t$. The solid lines are the simulation results based on the microstructure cell model and the strain gradient plasticity theory considering the grain size nonuniformity effect. For comparison, (d) shows the hardness-depth data for single crystal aluminum, where solid line is the theoretical modeling result based on the strain gradient plasticity theory.

comparison of Fig. 4(d) with Fig. 4(b) and (c), the SNCA material has a much higher hardness than single crystal aluminum, and the strip width of hardness-depth data of the SNCA material is much larger than that of the single crystal aluminum. The discrete feature of hardness-depth data of the single crystal aluminum can be taken as the effect due to the surface roughness.

The size effect and geometrical effect can be characterized by using a microstructure cell model and by adopting the strain gradient theory (Wei et al., 2003, 2004a). The grain size nonuniformity effect at a given distance $D$ can be described by introducing a measurable parameter, $\Psi$, which is defined as

$$
\Psi=\frac{t_{\max }-t}{t}=\frac{t-t_{\min }}{t},
$$

where $t$ is the average grain size along indenting direction at a given $D, t_{\max }$ and $t_{\min }$ are the maximum and minimum values of grain sizes at the given $D$, respectively. $\Psi$ 
describes the grain size nonuniformity, can be measured with the help of TEM observations.

\section{Theoretical modeling for the SNCA material}

Since the size effect cannot be simulated by using the conventional elastic-plastic theory, here the strain gradient plasticity theory combined with the microstructure cell models will be adopted to model and simulate the hardness-depth curves for the SNCA material. Fleck-Hutchinson's strain gradient plasticity flow theory (Fleck and Hutchinson, 1997; Wei and Hutchinson, 1997) will be adopted here. In the fundamental relations of the strain gradient plasticity flow theory, an additional lengthscale parameter, $L$ (SG theory), is included. At the macroscale, the strain gradient effect is very weak and can be neglected, and the strain gradient theory will be retrograded to the conventional elastic-plastic theory. However, at the microscale (micron or sub-micron), this effect is important. Usually $L$ is called the microscale length parameter. An overview on the microstructure cell models and the stain gradient plasticity flow theory will be briefly presented as follows.

\subsection{Microstructure cell models}

From observations of microstructure features of the SNCA (Fig. 1(d)-(f)) and analyses of the nanoindentation experiment, a microstructure cell models for indentation problem can be developed, as shown in Figs. 5 and 6 for the cases of non-penetrating and penetrating the grain boundaries by plastic zone, respectively.

\subsubsection{Non-penetrating the grain boundaries by plastic zone}

For the case of non-penetrating, i.e. when indent depth is smaller than grain size, or when grain boundary has a strong constraint on the plastic zone crossing, the plastic zone is always constrained within the indented grain region. The indented grain deforms plastically and the others deform elastically. The indented grain region can be treated with the elastic-plastic material described by the strain gradient plasticity theory. All other grain regions can be treated with an equivalent elastic medium described by a conventional elastic theory, and the equivalent elastic parameters (Young's modulus and Poisson ratio) can be estimated by using the Hill self-consistent theory (Hill, 1965). When the grain size is in the submicron scale (100 nm to $1 \mu \mathrm{m})$, the length scale is the same quantity level as the size-sensitive indent depth. The nanoindentation procedures and deformation phenomena can be described as follows: when indenter acts on a grain surface, as indent depth gradually increases, near the indenter tip the plastic slip zone size increases and expands to the grain boundary within the indented grain region. However, due to resistance of the grain boundaries, the plastic slip zone is constrained to the indented grain region. The other grains outside the indented grain region only undergo the elastic deformation. The total region the other grains occupy can be approximately treated with an equivalent uniform and isotropic elastic body. According to self-consistent theory (Hill, 

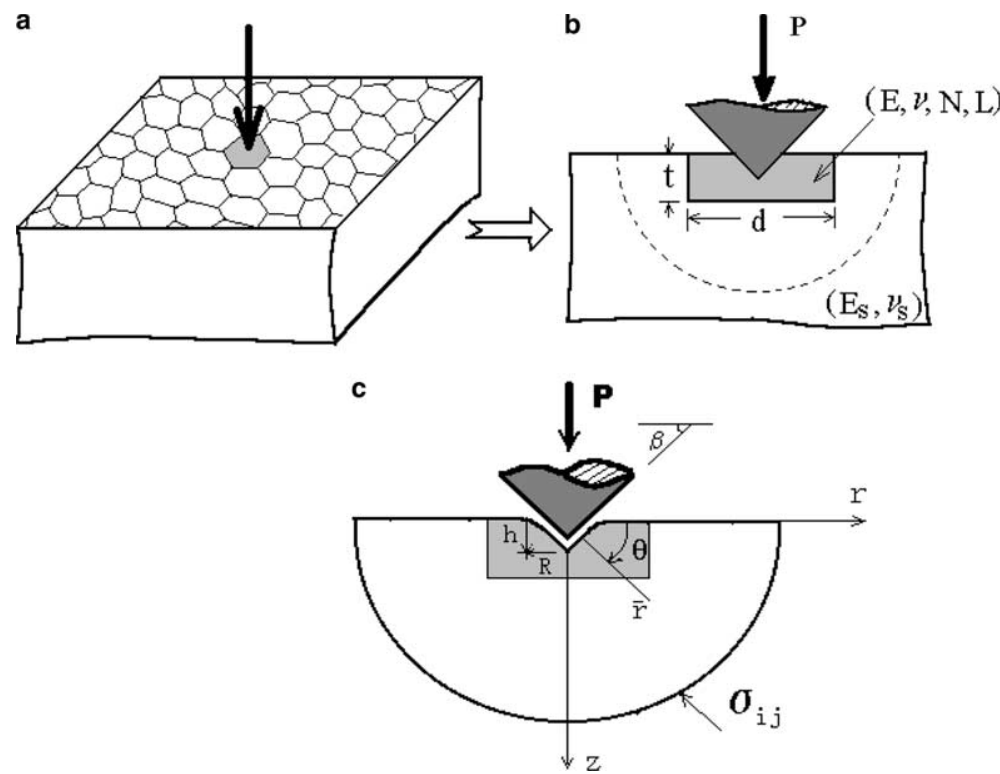

Fig. 5. The microstructure cell model for the case of non-penetrating grain boundaries by plastic zone.
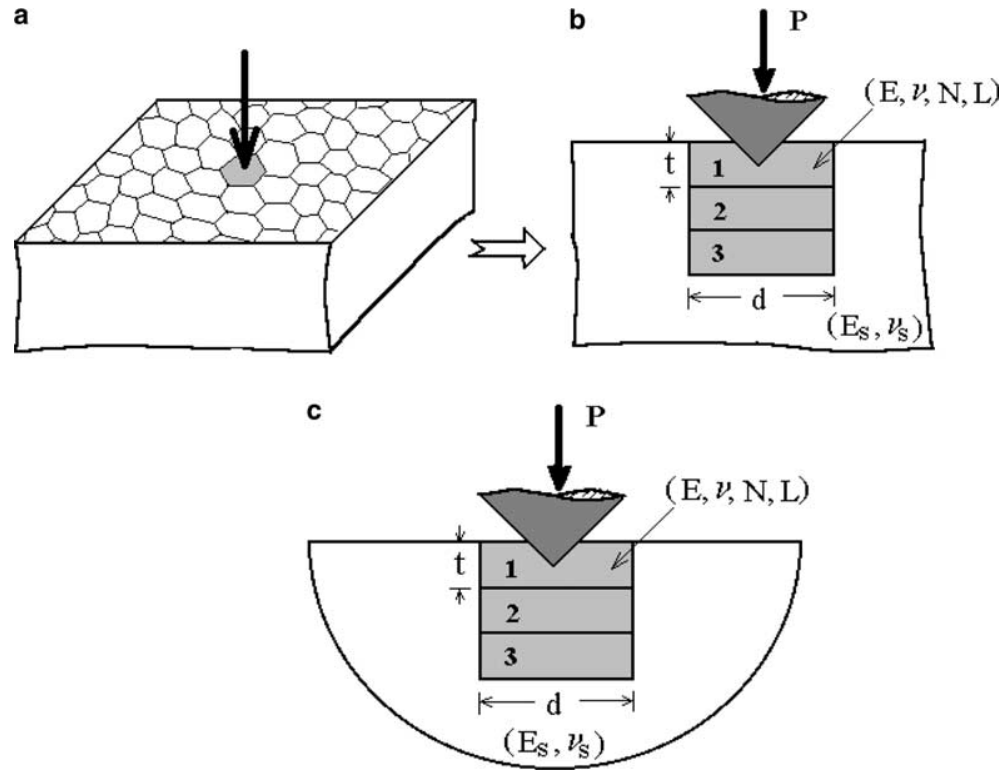

Fig. 6. The microstructure cell model for the case of the plastic zone penetrating grain boundaries.

1965), equivalent Young's modulus and Poisson's ratio can be found. The related problem of the self-consistent method can be summarized as follows: for the equivalent modulus problem of a polycrystal material when grain shape is a cylinder, 
consider that a cylinder is located in an infinite equivalent elastic body, solve the problem to find the equivalent modulus. Obtained Young's modulus for the equivalent elastic body is 0.671 times the average Young's modulus of single crystal grain. If one considers the grain shape as an ellipsoid, Young's modulus of the equivalent elastic body is 0.614 times the average Young's modulus of single crystal grain. From previous research, the size effect result was not sensitive to the coefficient (Wei et al., 2003). For the indented grain deformed plastically, the grain can be approximately treated with a uniform and isotropic elastic-plastic material (for the conventional FCC metals, this simplification is reasonable), simultaneously the strain gradient effect is considered. The simplified sketches are given in Fig. 5(a) and (b). Considering the cylinder grain shape, the problem can be further simplified to an axisymmetrical problem, as described by Fig. 5(c). At the remote boundary, an elastic field (a solution of a point load acting on the semi-infinite body surface) (see Timoshenko and Goodier, 1970) is exerted.

\subsubsection{Plastic zone penetrating the grain boundaries}

For the case of plastic zone penetrating grain boundaries, the simplified cell model is described by Fig. 6. Differing from the model shown in Fig. 5, a condition of the plastic zone penetrating grain boundaries is presented. The plastic zone is constrained by the grain boundaries, and the limit constraint strength can be described by the critical effective plastic strain. Increasing indentation load further, when effective plastic strain at the point on the grain boundaries reaches the critical value, the plastic zone will penetrate the grain boundaries and extend to the neighboring grains. In the model, as shown in Fig. 6, as long as plastic zone extends to a new grain region, the new grain region will be absorbed into the elastic-plastic material region. For example, increasing the indentation load when plastic zone extends to other two grain regions, the model evolution is described in Fig. 6.

Consider that when effective plastic strain at grain boundary is over a critical value, $\bar{\varepsilon}_{\mathrm{P}}^{\mathrm{c}}$, the plastic zone will cross the grain boundary and extend to neighboring grain region. The condition of the plastic slip zone across the grain boundary can be expressed as

$$
\left.\bar{\varepsilon}_{\mathrm{P}}\right|_{\Gamma}=\bar{\varepsilon}_{\mathrm{P}}^{\mathrm{c}},
$$

where $\Gamma$ stands for the grain boundary. By using the critical value of the effective plastic strain, one can further characterize the grain boundary features, such as hardening, weakening, damage, etc.

\subsection{Strain gradient plasticity flow theory}

In the present research, Fleck and Hutchinson strain gradient plasticity flow theory (Fleck and Hutchinson, 1997; Wei and Hutchinson, 1997) will be adopted. An overview on the theory is presented as follows (Wei and Hutchinson, 1997). 
Constitutive equations for strain gradient plasticity flow theory:

$$
\begin{aligned}
\dot{\sigma}_{i j}= & {\left[\bar{L}_{i j k l}-\frac{9 \mu}{(3+H) \sigma_{\mathrm{e}}^{2}} \sigma_{i j}^{\prime} \sigma_{k l}^{\prime}\right] \dot{\varepsilon}_{k l}+\left[-\frac{6 E\left(L_{\mathrm{e}} / L\right)^{2}}{(3+H) \sigma_{\mathrm{e}}^{2}} \sigma_{i j}^{\prime} \sum_{I=1}^{3}\left(\frac{k_{\mathrm{e}}^{(I)}}{k^{(I)}}\right)^{2} \tau_{k l m}^{(I)}\right] \dot{\eta}_{k l m}, } \\
\dot{\tau}_{i j k}= & {\left[-\frac{6 E\left(L_{\mathrm{e}} / L\right)^{2}}{(3+H) \sigma_{\mathrm{e}}^{2}} \sigma_{m n}^{\prime} \sum_{I=1}^{3}\left(\frac{k_{\mathrm{e}}^{(I)}}{k^{(I)}}\right)^{2} \tau_{i j k}^{(I)}\right] \dot{\varepsilon}_{m n} } \\
& +\left[J_{i j k l m n}-\frac{8(1+v) E\left(L_{\mathrm{e}} / L\right)^{4}}{(3+H) \sigma_{\mathrm{e}}^{2}} \sum_{I=1}^{3}\left(\frac{k_{\mathrm{e}}^{(I)}}{k^{(I)}}\right)^{2} \tau_{i j k}^{(I)} \sum_{J=1}^{3}\left(\frac{k_{\mathrm{e}}^{(J)}}{k^{(J)}}\right)^{2} \tau_{l m n}^{(J)}\right] \dot{\eta}_{l m n},
\end{aligned}
$$

where, $\sigma_{\mathrm{e}}=\sqrt{3 \sigma_{i j}^{\prime} \sigma_{i j}^{\prime} / 2}$ is conventional effective stress, $\mu=E /[2(1+v)]$ is shear modulus, $H$ is effective hardening coefficient and can be expressed as

$$
H=\left(\frac{\Sigma}{\sigma_{\mathrm{e}}}\right)^{2}\left[1+\frac{2 E L_{\mathrm{e}}^{2}}{h \Sigma^{2} L^{4}} \sum_{I=1}^{3} \frac{\left(k_{\mathrm{e}}^{(I)}\right)^{2}}{\left(k^{(I)}\right)^{4}} \tau_{i j k}^{(I)} \tau_{i j k}^{(I)}\right] \frac{h}{\mu},
$$

$h$ is plastically hardening modulus, and it can be expressed as

$$
\begin{aligned}
& \frac{1}{h}=0 \quad \text { for } \Sigma \leqslant \sigma_{\mathrm{Y}}, \\
& \frac{1}{h}=\frac{1}{E}\left[\frac{1}{N}\left(\frac{\Sigma}{\sigma_{\mathrm{Y}}}\right)^{(1-N) / N}-1\right] \quad \text { for } \Sigma>\sigma_{\mathrm{Y}},
\end{aligned}
$$

$N$ is strain hardening index for power-law hardening materials, $\sigma_{\mathrm{Y}}$ is yield strength, $\Sigma$ is effective stress, and is expressed as

$$
\Sigma^{2}=3 \sigma_{i j}^{\prime} \sigma_{i j}^{\prime} / 2+L^{-2} \sum_{I=1}^{3}\left(k^{(I)}\right)^{-2} \tau_{i j k}^{(I)} \tau_{i j k}^{(I)}
$$

Elastic modulus tensors give by

$$
\begin{aligned}
& \bar{L}_{i j k l}=\frac{E}{2(1+v)}\left[\delta_{i k} \delta_{j l}+\delta_{i l} \delta_{j k}+\frac{2 v}{(1-2 v)} \delta_{i j} \delta_{k l}\right], \\
& J_{i j k l m n}=2 E L_{\mathrm{e}}^{2} \sum_{I=1}^{4} k_{\mathrm{e}}^{(I) 2} T_{i j k l m n}^{(I)},
\end{aligned}
$$

and their reverse relations are

$$
\begin{aligned}
& M_{i j k l}=\bar{L}_{i j k l}^{-1}=\frac{1}{E}\left[\frac{1+v}{2}\left(\delta_{i k} \delta_{j l}+\delta_{i l} \delta_{j k}\right)-v \delta_{i j} \delta_{k l}\right], \\
& K_{i j k l m n}=J_{i j k l m n}^{-1}=\frac{1}{2 E L_{\mathrm{e}}^{2}} \sum_{I=1}^{4}\left(k_{\mathrm{e}}^{(I)}\right)^{-2} T_{i j k l m n}^{(I)} ;
\end{aligned}
$$

where $T_{i j k l m n}^{(I)},(I=1,4)$ are the strain gradient projection tensors, their expressions have been derived by Wei and Hutchinson (1997). $L k^{(I)}=L_{I}(I=1,3)$ is microscale 
length parameters, $0 \leqslant k^{(I)} \leqslant 1$ is length scale ratio, dimensionless. From discussions by Fleck and Hutchinson (1997), usually, $k^{(I)}$ (or $k_{\mathrm{e}}^{(I)}$ ) follows from the two types:

(1) For couple stress case (CS):

$$
k^{(1)}=0, \quad k^{(2)}=1 / 2, \quad k^{(3)}=\sqrt{5 / 24} .
$$

(2) For strain gradient general case (SG):

$$
k^{(1)}=1, \quad k^{(2)}=1 / 2, \quad k^{(3)}=\sqrt{5 / 24} .
$$

In the present analysis, take $k^{(4)}=1 / 2, L_{\mathrm{e}} / L=0.5$, referring to Wei and Hutchinson (1997), and adopt the SG theory (see (9)). $L$ is microscale length parameter in strain gradient plasticity.

\section{Simulations for the SNCA material}

In Section 2, we have obtained the nano-indentation experimental results which include the hardness-depth data relations by using the randomly selected load point method when indent depths are smaller than grain sizes, and the loading-/hardnessdepth curves by using the continuous stiffness method when indenter (or plastic zone) penetrates the grain boundaries. In the present section, using the microstructure cell models and the strain gradient plasticity flow theory models the above discussed two kinds of the nanoindentation experimental processes by adopting finite element method. The element adopted here is the iso-parametrical displacement element with nine nodes (Wei and Hutchinson, 1997).

\subsection{For the case of non-penetrating the grain boundaries by plastic zone}

The microstructure cell model is shown in Fig. 5. In the simplified model, besides the conventional material parameters, there are three additional governing parameters: the grain size, the micro-scale parameter $L$ and the grain size nonuniformity factor $\Psi$. Through dimensional analysis, the dimensionless parameter relationship of hardness-depth curve can be expressed as

$$
\frac{H}{3 \sigma_{\mathrm{Y}}}=f\left(\frac{h}{L} ; \frac{d}{2 L}, \frac{t}{L}, \frac{E}{\sigma_{\mathrm{Y}}}, \frac{E_{\mathrm{s}}}{E}, v, v_{\mathrm{s}}, N, \Psi\right) .
$$

Through finite element simulation, the detailed relationship of hardness-depth is obtained and plotted in Fig. 7 for several parameter values and for $2 t / d=0.2$, a flat grain case as discussed in Section 2. In Fig. 7, six cases of grain sizes, $t / L=0.364,0.5$, $0.7,1.0,1.5$ and 10, are plotted, corresponding to the different grain size effects. The hardness-depth curve is with a "U-type" feature as we expect and experiment shows in Section 2. When indent depth is small, size effect from length scale parameter in the strain gradient theory dominates. With increasing indent depth, both size effect and hardness value decrease, and the hardness curve goes down through a minimum value, then goes up due to the plastic zone constrained by grain boundaries, i.e., the 


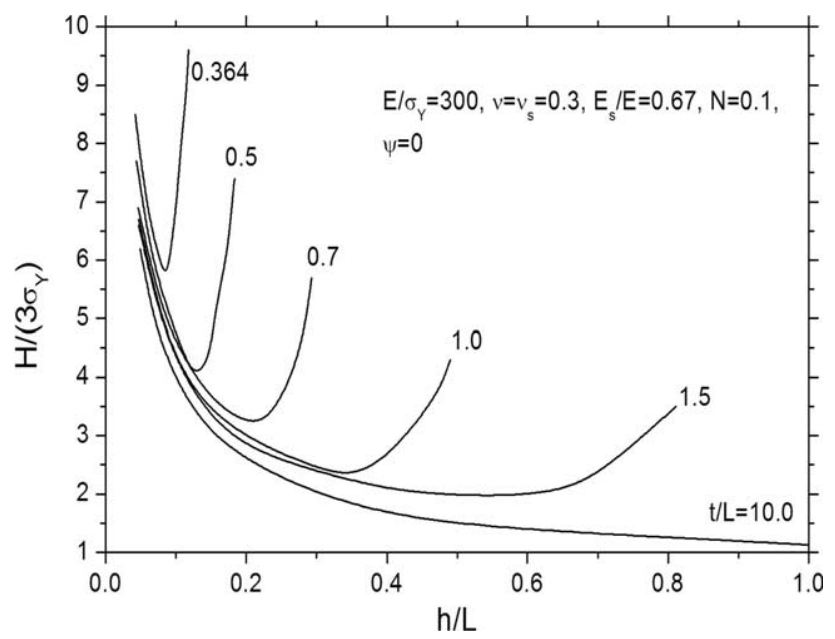

Fig. 7. Variations of the normalized hardness as a function of the normalized indent depth for several grain size values based on the microstructure cell model (see Fig. 5). The "U-type" simulated curves are seen from figure.

geometrical effect dominates. From Fig. 7, for a given $L$ value, with increasing grain size, the hardness-depth curve goes down and the geometrical effect considerably decreases.

It is worth noting that the results shown in Fig. 7 plotted for the case of $\Psi=0$ (not considering the grain size nonuniformity effect) can be easily used to analyze the general case of $\Psi \neq 0$. Actually, the grain size, $t$, can be taken as the average grain size for a given $D$ (distance away from the NC surface). For the given values of $(t, \Psi)$, one can obtain two corresponding curves of hardness-depth relation, corresponding to the upper bound (for grain size $t_{\min }$ ) and lower bound (for grain size $t_{\max }$ ), respectively. (For the case of $\Psi=0$, both bound curves become a unique one). Using these solutions to simulate the experimental data given in Fig. 4, the simulating results are also shown in Fig. 4. The experimental data strip of hardness-depth relation can be interpreted effectively by the grain size nonuniformity effect. It is worth pointing out that in Fig. 4, the strain gradient length parameter $L$ is taken as a fixed value in all cases, $1 \mu \mathrm{m}$, which is determined by using the strain gradient plasticity theory to model the single crystal aluminum case as shown in Fig. 4(d), so the length scale $L$ can be fully taken as a material parameter instead of a simulation parameter.

\subsection{For the case of plastic zone penetrating the grain boundaries}

In Section 2, we have discussed the experimental hardness-depth curves, as shown in Figs. 2 and 3, obtained by using the continuous stiffness method. Obviously, in this case the indenter (or plastic zone) has penetrated the grain boundaries. The hardness-depth curves are with a "waterfall-type" character. In the present subsection, we adopt the microstructure cell model (see Fig. 6) and the strain gradient 
theory to model the hardness-depth relations. In the simplified model, besides the conventional material parameters, there are four governing parameters: the grain size, the micro-scale parameter $L$, the grain size nonuniformity factor $\Psi$, as well as the strength criterion parameter of the plastic zone penetrating grain boundaries, $\bar{\varepsilon}_{\mathrm{P}}^{\mathrm{c}}$. Through dimensional analysis, the dimensionless parameter relation of the hardness-depth curve can be expressed as

$$
\frac{H}{3 \sigma_{\mathrm{Y}}}=f\left(\frac{h}{L} ; \frac{d}{2 L}, \frac{t}{L}, \frac{E}{\sigma_{\mathrm{Y}}}, \frac{E_{\mathrm{s}}}{E}, v, v_{\mathrm{s}}, N, \Psi, \bar{\varepsilon}_{\mathrm{P}}^{\mathrm{c}}\right) .
$$

Through finite element simulation, the detailed relationship between hardness and depth are obtained and plotted in Fig. 8 for several parameter values and for two cases: (1) $2 t / d=0.2,2 t_{2} / d=0.08,2 t_{3} / d=0.12$ and (2) $2 t / d=0.4,2 t_{2} / d=0.2$, where $t_{2}$ and $t_{3}$ are the sizes of the grains absorbed into the plastic zone in the second and the third order, referring to Fig. 6. From Fig. 8, the hardness-depth curves for both cases are with the "waterfall-type" feature, the trend is similar to that by experimental measurement. The results show that with increasing the indent depth, the size effect decreases and the geometrical effect increases due to plastic zone constrained by grain boundaries. When the constraining characterized quantity (effective plastic strain) attains a critical value at some points on the grain boundaries, the plastic zone sharply extends to the neighboring grain region, correspondingly, the hardness value decreases. With further increasing the indent depth, the plastic zone expands within the new and enlarged region, reaches a new grain boundary, and is

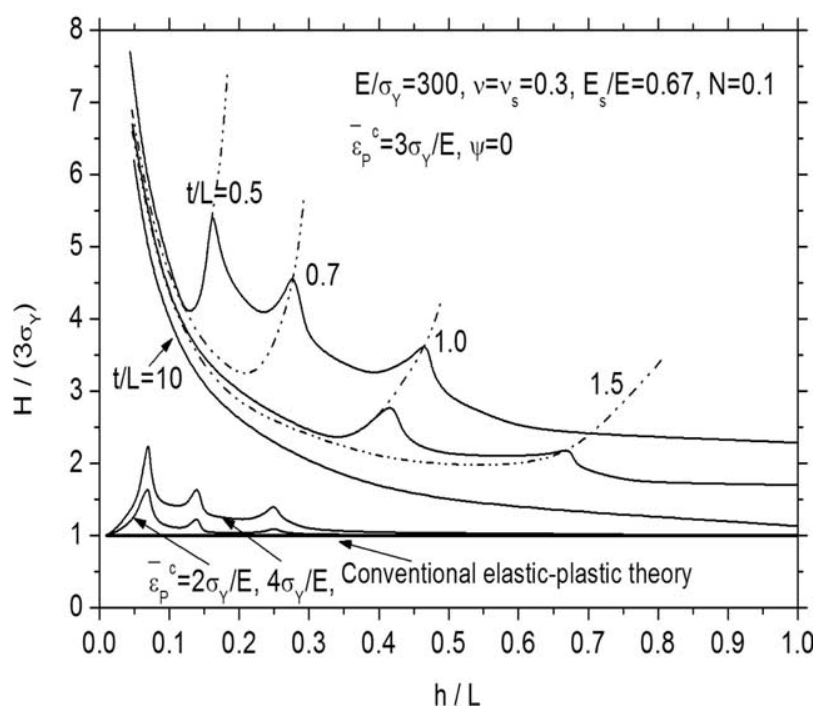

Fig. 8. Variations of the normalized hardness as a function of the normalized indent depth for several grain size values based on the microstructure cell model (see Fig. 6) considering the plastic zone penetrating the grain boundaries. The "waterfall-type" simulated curves are seen from figure. The results based on the conventional elastic-plastic theory are also shown for comparison. 
then constrained by the new grain boundary, and the hardness curve goes up again. From Fig. 8, comparing the results of the case (1) and case (2), since grain size ( $t$ value) near the specimen surface for the case (1) is smaller than that for the case (2), the "waterfall-type" hardness curve for the case (1) is higher than that for the case (2).

The results shown in Fig. 8 can be used to simulate the experimental results for $\Psi \neq 0$ (considering the grain size nonuniformity effect) like Fig. 2. The difference between the grain sizes on the specimen surface causes the different hardness curves.

Using the microstructure cell model and the strain gradient plasticity theory simulates the experimental results given in Fig. 3. The simulated results are plotted and compared with experimental results as also shown in Fig. 3. Obviously, using the microstructure cell model and the strain gradient plasticity theory to simulate the nanoindentation test for the SNCA material is effective.

\section{Concluding remarks}

The mechanical behaviors of the SNCA material manufactured by using the USP method have been investigated through microscopy observation and measurement, nanoindentation experiments and the theoretical modeling and simulations. By using the microstructure cell models and the strain gradient plasticity theory, the characteristics of the hardness-depth curves for the SNCA material, such as the "U-type" and "waterfall type" features, have been modeled and simulated successfully. By introducing a measurable parameter to characterize the grain size nonuniformity effect, the discrete features of the hardness-depth relations have been described, and the microscale length parameter in the strain gradient theory can be taken as a universal material parameter instead of the simulating parameter. The length scale parameter is uniquely determined through applying the strain gradient plasticity theory to the modeling of the single crystal material. In the present research, the grain boundary mechanical properties have been characterized by introducing a criterion parameter, a critical effective plastic strain.

It is worth pointing out that if we adopt the conventional elastic-plastic theory instead of the strain gradient theory to model the hardness-depth relationship for the SNCA material, we have found that the obtained hardness results (see Fig. 8) are too low to compare with experimental data (see Fig. 2). Even through increasing the penetrating strength of the grain boundary, the hardness value is still very low.

Through microstructure observations for the SNCA material, we have found that near the NC surface the grain size is very small (several nanometers). However, when grain size is smaller than submicron, it is difficult to confirm the effectiveness of both the microstructure cell models and the strain gradient plasticity theory, although the strain gradient theory can be applied to a smaller scale to compare with the conventional elastic-plastic theory. Therefore, when grain size is smaller than submicron, the simulated results can only be taken as a rough estimation for the SNCA material. 


\section{Acknowledgements}

This work was supported by the National Natural Science Foundation of China through Grant 19925211 and by the "Bai Ren" Project of the Chinese Academy of Sciences. Authors also acknowledge the support of the National Natural Science Foundation of China through Key Project (No. 10432050) and the Oversea Co-operation Grant (No. 10428207).

\section{References}

Abu Al-Rub, R.K., Voyiadjis, G.Z., 2004. Analytical and experimental determination of the material intrinsic length scale of strain gradient plasticity theory from micro- and nano-indentation experiments. International Journal of Plasticity 20, 1139-1182.

Acharya, A., Bassani, J.L., 2000. Lattice incompatibility and a gradient theory of crystal plasticity. J. Mech. Phys. Solids 48 (8), 1565-1595.

Aifantis, E.C., 1984. On the microstructural origin of certain inelastic models. Trans ASME J. Eng. Mater. Tech. 106, 326-330.

Begley, M., Hutchinson, J.W., 1998. The mechanics of size-dependent indentation. J. Mech. Phys. Solids 46, 1029.

Bonfoh, N., Lipinski, P., Carmasol, A., Tiem, S., 2004. Micromechanical modeling of ductile damage of polycrystalline materials with heterogeneous particles. Int. J. Plasticity 20, 85-106.

Brunig, M., Ricci, S., 2005. Nonlocal continuum theory of anisotropically damaged metals. Int. J. Plasticity 21, 1346-1382.

Chen, S.H., Wang, T.C., 2000. A new hardening law for strain gradient plasticity. Acta Mater. 48, 3997.

Chen, S.H., Wang, T.C., 2002. A new deformation theory with strain gradient effects. Int. J. Plasticity 18, 971-995.

Fleck, N.A., Hutchinson, J.W., 1997. Strain gradient plasticity. In: Hutchinson, J.W., Wu, T.Y. (Eds.), Advances in Applied Mechanics vol. 33, p. 295.

Fleck, N.A., Muller, G.M., Ashby, M.F., Hutchinson, J.W., 1994. Strain gradient plasticity: theory and experiments. Acta Metall. Mater. 42, 475-487.

Gao, H., Huang, Y., Nix, W.D., Hutchinson, J.W., 1999. Mechanism-based strain gradient plasticity-I. Theory J. Mech. Phys. Solids 47, 1239.

Gerberich, W.W., Tymiak, N.I., Grunlan, J.C., Horstemeyer, M.F., Baskes, M.I., 2002. Interpretations of indentation size effects. J. Appl. Mech. 69, 433-442.

Hill, R., 1965. Continuum micro-mechanics of elastoplastic polycrystals. J. Mech. Phys. Solids $13,89$.

Horstemeyer, M.F., Baskes, M.I., Plimpton, S.J., 2001. Length scale and time scale effects on the plastic flow of FCC metals. Acta Mater. 49, 4363-4374.

Huang, Y., Qu, S., Hwang, K.C., Li, M., Gao, H., 2004. A conventional theory of mechanism-based strain gradient plasticity. Int. J. Plasticity 20, 753-782.

Hwang, K.C., Jiang, H., Huang, Y., Gao, H., 2003. Finite deformation analysis of mechanism-based strain gradient plasticity: torsion and crack tip field. Int. J. Plasticity 19, 235-251.

Lu, K., Lu, J., 1999. Surface nanocrystallization (SNC) of metallic materials-presentation of the concept behind a new approach. J Mater. Sci. Technol. 15, 193.

Ma, Q., Clarke, D.R., 1995. Size dependent hardness of silver single crystals. J. Mater. Res. 10, $853-863$.

Ma, X.L., Wang, W., Yang, W., 2003. Simulation for surface self-nanocrystallization under shot peening. Acta Mechanica Sinica 19 (2), 172.

McElhaney, K.W., Vlassak, J.J., Nix, W.D., 1998. Determination of indenter tip geometry and indentation contact area for depth-sensing indentation experiments. J. Mater. Res. 13, 1300-1306.

Saczuk, J., Hackl, K., Stumpf, H., 2003. Rate theory of nonlocal gradient damage-gradient viscoinelasticity. Int. J. Plasticity 19, 675-706. 
Stolken, J.S., Evans, A.G., 1998. A microbend test method for measuring the plasticity length scale. Acta Mater. 46, 5109-5115.

Taylor, M.B., Zbib, H.M., Khaleel, M.A., 2002. Damage and size effect during superplastic deformation. Int. J. Plasticity $18,415-442$.

Timoshenko, S.P., Goodier, J.N., 1970. Theory of elasticity, Third ed. McGraw-Hill Inc, New York, p. 401.

Valiev, R.Z., Islamgaliev, R.K., 1999. Enhanced superplasticity of ultrafine-grained alloys processed by severe plastic deformation. Materials Science Forum 304, 39.

Valiev, R.Z., Korznikov, A.V., Mulyukov, R.R., 1993. Structure and properties of ultrafine-grained materials produced by severe plastic deformation. Mater. Sci. Eng. A 168, 141.

Valiev, R.Z., Ivanisenko, Y.V., Rauch, E.F., Baudelet, B., 1996. Structure and deformation behavior of armco iron subjected to severe plastic deformation. Acta Mater. 44, 4705.

Valiev, R.Z., Islamgaliev, R.K., Alexandrov, I.V., 2000. Bulk nanostructured materials from severe plastic deformation. Progr. Mater. Sci. 45, 103.

Wei, Y., 2001. Particulate size effects in the particle-reinforced metal matrix composites. Acta Mechanica Sinica 17 (1), 45-58.

Wei, Y., Hutchinson, J.W., 1997. Steady-state crack growth and work of fracture for solids characterized by strain gradient plasticity. J. Mech. Phys. Solids 45 (8), 1253.

Wei, Y., Hutchinson, J.W., 2003. Hardness trends in micron scale indentation. J. Mech. Phys. Solids 51, 2037-2056.

Wei, Y., Wang, X., Wu, X., Bai, Y., 2001. Theoretical and experimental researches of size effect in microindentation test. Science in China (Series A) 44 (1), 74.

Wei, Y., Wang, X., Zhao, M., Cheng, C.M., Bai, Y., 2003. Size effect and geometrical effect of solids in micro-indentation test. Acta Mechanica Sinica 19 (1), 59.

Wei, Y., Zhu, C., Wu, X., 2004a. Micro-scale mechanics of the surface-nanocrystalline Al-alloy material. Science in China (Series G) 47 (1), 86-100.

Wei, Y., Wang, X., Zhao, M., 2004b. Size effect measurement and characterization in nanoindentation test. J. Mater. Res. 19 (1), 208-217.

Wu, X., Tao, N., Hong, Y., 2002. Microstructure and evolution of mechanically-induced ultrafine grain in surface layer of Al-alloy subjected to USSP. Acta Mater. 50, 2075.

Xue, Z., Huang, Y., Li, M., 2002. Particle size effect in metallic materials: a study by the theory of mechanism-based strain gradient plasticity. Acta Mater. 50, 149-160.

Zhu, C., 2002. Microstructure characteristics and microscale mechanics of the surface-nanocrystalline Alalloy material, Master degree thesis. Institute of Mechanics, Chinese Academy of Sciences, June (in Chinese). 